\title{
Letter Response to "Investigating the Sources of Heterogeneity in Systematic Review of Recurrence Rate after Hemi-Thyroidectomy for Low-Risk Well-Differentiated Thyroid Cancer"
}

\author{
Samuel Chan ${ }^{\mathrm{a}}$ Katarina Karamali ${ }^{\mathrm{a}}$ Anna Kolodziejczyk $^{\mathrm{a}}$ Georgio Oikonomou $^{\mathrm{a}}$ \\ John Watkinson ${ }^{b}$ Vinidh Paleric lain J. Nixon ${ }^{d}$ Dae Kim ${ }^{a}$ \\ ${ }^{a}$ Department of Ear, Nose and Throat Surgery, Lanesborough Wing, St George's Hospital, London, UK; ${ }^{b}$ Department \\ of Ear, Nose and Throat Surgery, GOSH, London, UK; ' ${ }^{\text {Head }}$ and Neck Unit, The Royal Marsden Hospital, London, UK; \\ ${ }^{\mathrm{d}}$ Department of Ear, Nose and Throat Surgery, NHS Lothian, Lauriston Building, University of Edinburgh, Edinburgh, \\ UK
}

Dear Editor,

In our recently published study, we have systematically reviewed the current literature regarding recurrence rates in well-differentiated thyroid cancer after hemi-thyroidectomy (HT) [1]. Through meta-analysis of available data, we have shown that the pooled recurrence rate after HT in all included studies, as well as a subset of these studies that define a low-risk cohort of patients, is approximately $9 \%$ in both groups. We found this rate to be marginally but statistically significantly higher compared to total thyroidectomy (TT).

We are grateful that Sahovaler et al. [2] have been able to use the data we have displayed to reproduce our findings of a statistically significant increase in recurrence rate after HT compared to TT, whilst achieving reduced study heterogeneity through their own grouping strategy. Heterogeneity is the bugbear of systematic reviews, especially those that do not involve RCTs, and our colleagues have reiterated an important issue that we also highlighted in our paper. We accept that appropriate measures should be taken to minimize heterogeneity.

We agree that if grouping is specifically confined to tumor sizes 1-4 cm, Hay et al. [3] would be in a separate group and acknowledge the higher effect weighting of the karger@karger.com

www.karger.com/etj

Karger" study. However, the issue of how to define low-risk disease is a debate that precedes the publication by Hay et al. [3]. Choosing to include or exclude varying scoring systems is contentious and may lead to bias in itself. Additionally, in reducing study heterogeneity by excluding Hay et al. [3] from analysis, the pooled number of cases is reduced from 3,332 to 1,694 (a 49\% reduction), and analysis is less able to achieve significance in small but clinically important differences.

It is our opinion that in the absence of a single unifying low-risk classification system that could be applied post hoc to the raw data sets from these various studies, it would be important to include all cohorts classified as low-risk according to their own recognized classification systems. This we think gives a more balanced picture at the expense of statistical study heterogeneity.

The letter from Sahovaler et al. further emphasizes the need for prospectively collected data within a randomized controlled setting comparing the outcomes of HT and TT. We are aiming to address this very deficit in our upcoming National Cancer Research Institute funded randomized controlled trial called the hemithyroidectomy or total thyroidectomy in low-risk thyroid cancer trial (HoT trial). In the age of treatment de-escalation, par-
(C) 2021 European Thyroid Association

Published by S. Karger AG, Basel 
ticularly in this cohort of low-risk thyroid cancer patients, clinicians and patients alike need robust data to demonstrate that HT is non-inferior to TT in terms of outcomes such as disease recurrence.

\section{Conflict of Interest Statement}

The authors have no conflicts of interest to declare.

\section{Funding Sources}

Not applicable.

\section{Author Contributions}

S.C. wrote the response in consultation with all other co-authors. Final response letter was reviewed and approved by all senior co-authors prior to submission.

\section{References}

1 Chan S, Karamali K, Kolodziejczyk A, Oikonomou G, Watkinson J, Paleri V, et al. Systematic review of recurrence rate after hemithyroidectomy for low-risk well-differentiated thyroid cancer. Eur Thyroid J. 2020; 9(2):73-84.
2 Sahovaler Axel, Ferrari Marco, Athayde Jonathan, Mendez Adrian, Fung Kevin, Yoo John, et al. Investigating the Sources of Heterogeneity in Systematic Review of Recurrence Rate after Hemithyroidectomy for Low-Risk WellDifferentiated Thyroid Cancer. Eur Thyroid J. 2020;1-4.
3 Hay ID, Grant CS, Bergstralh EJ, Thompson GB, van Heerden JA, Goellner JR. Unilateral total lobectomy: is it sufficient surgical treatment for patients with AMES low-risk papillary thyroid carcinoma? Surgery. 1998;124(6): 958-64. discussion 64-6. 